\title{
Neuromodulation: a review of deep brain stimulation devices for the treatment of Parkinson's disease
}

\author{
Keywords: stimulation, epilepsy, neuromodulators
}

Abbreviations: DBS, deep brain stimulation; SCS, spinal cord stimulator, IPG, implantable pulse generator

\section{Introduction}

A neuromodulation device is a device that is used to directly act upon a patient's nerves to deliver electrical or pharmaceutical stimulation for therapeutic purposes. ${ }^{1}$ Neuromodulation is an ancient practice that started with the Ancient Romans who used torpedo fish to deliver electric shocks to alleviate pain. ${ }^{1}$ However, neuromodulation devices and sciences began to take shape as they are known today it in the 1960's and 1970's. Neuromodulation has a checkered past, especially when considering Deep Brain Stimulation (DBS). ${ }^{1}$ Originally, DBS was used for ablation surgeries to help patients who suffered from epilepsy. It was not until 2002 that DBS was approved by the FDA to treat Parkinson's Disease. ${ }^{1}$

There are many indications for the use of neuromodulation. One problem that patients commonly face is chronic pain and there is a wide market for chronic pain management. ${ }^{2}$ Chronic pain is a cumbersome problem for the patient and the health care system to combat; especially in light of a large movement to avoid prescribing opioids. ${ }^{2}$ A Spinal Cord Stimulator (SCS) is a neuromodulation device that can be used to treat chronic pain. ${ }^{3}$ SCS is a tool that stimulates the basal ganglion with adjustable bursts and high frequency stimulation that can target specific sources of pain. The implantable leads are adjustable and allow the device to zero in on the specific synapse that is causing pain; this disrupts the messaging of that synapse with electrical impulses. ${ }^{2}$

Sacral Nerve Stimulation (SNS) is another form of neuromodulation that is used to treat issues with the pelvic floor such as urge continence or severe constipation. ${ }^{3,4}$ SNS is an attractive option for patients who suffer from pelvic floor issues who cannot tolerate the usual interventions. As with most neuromodulators it is made up of three parts, the pulse generator, an extension cable, and the electrodes. ${ }^{3}$ The electrodes are typically implanted in the sacral foramens and the pulse generator is usually permanently implanted in to the buttocks. ${ }^{3}$ After implantation, the SNS can be programmed to help stimulate the pelvic floor voluntarily when the patient is experiencing symptoms. ${ }^{3}$ The focus of this paper will be on the way DBS is used to treat PD. Like SCS, DBS can, and has, been used to manage pain. ${ }^{4}$ Traditionally, electrodes are surgically implanted into the brain of a patient; the electrodes are connected to an impulse generator via extensions. ${ }^{4}$ Magnetic Resonance Imaging can be used to help healthcare providers place the electrodes in parts of the brain that have the best potential for desired clinical outcomes. ${ }^{4}$

\section{Parkinson's disease}

\section{Disease definition}

Parkinson's Disease is a disease that is caused from extremely

\author{
Volume 8 Issue I - 202 I \\ Nicholas Noli,' Marguerite Jackson,' Tawil \\ Bill1,2 \\ 'Department of Biotechnology and Bioinformatics, California \\ State University, USA \\ ${ }^{2}$ Department of Bioengineering, University of California Los \\ Angeles, USA
}

Correspondence: Bill Tawil, Department of Bioengineering, UCLA School of Engineering, 420 Westwood Plaza, Room 5121, Engineering V. P.O. Box: 951600, Los Angeles, CA 90095-1600, USA, Fax (310) 794-5956, Email bill.tawi@csuci.edu

Received: January 17, 202I | Published: February II, 202 I

low levels of dopamine neurons in the brain, specifically in the Substantia nigra. ${ }^{5}$ A patient with Parkinson's Disease typically experiences involuntary tremors in their hands, increasing stiffness of movement, bradykinesia, as well as gate and balance problems. ${ }^{5}$ Patients are typically diagnosed later in life when they are in their 50 's or 60 's, but lead a quintessential normal life up to the point when they start exhibiting symptoms. ${ }^{5}$ When patients are affected, mundane tasks, like pouring liquids, become impossible and many become housebound if left untreated. About one million Americans are affected by Parkinson's Disease and treatments of the disease can provide patients with a means to access a normal quality of life with the disease. ${ }^{5}$

In a healthy brain, the Substantia nigra is home to many dopamine neurons. ${ }^{6}$ Dopamine is an important neurotransmitter that contributes to normal functioning of movement, sleep, mood, and cognition, among other aspects. ${ }^{6}$ A lack of dopamine neurons in the Substantia nigra exhibits itself in the hallmark symptoms of Parkinson's disease such as: rigidity, resting tremors, and postural issues. ${ }^{7}$ The dopamine activity disruption in the Substantia nigra can be attributed to a misfolded protein called alpha synuclein and an aggregate of alpha synuclein is called a Lewy body. ${ }^{8}$ This accumulation of Lewy bodies critically disrupt the normal dopamine activity in the Substantia nigra and patients with Parkinson's Disease begin to exhibit the classic symptoms of Parkinson's Disease. ${ }^{7}$ The origin of the Lewy bodies is not fully understood, but can form in the gut when exposed to certain chemicals. ${ }^{7}$ Parkinson's disease is largely idiopathic. ${ }^{8}$ However, there are some factors that directly relate to the development of Parkinson's disease. One of these factors is age, as the disease does not usually develop until later in a person's life. ${ }^{8}$ Exposure to certain chemicals 
can also increase a person's risk of developing Parkinson's. ${ }^{8}$ In some cases, malformed alpha synucleins can begin to form in the gut when a patient is exposed to certain chemicals. ${ }^{8}$ However, the exact role that age, genes, or environmental factors in the development of Parkinson's Disease remains elusive.

\section{Classical treatments: drug interventions}

The most classic drug a patient with PD would take is levodopa. ${ }^{9}$ The aim of medication is to control a patient's "Off" time while avoiding the induction of debilitating dyskinesia. ${ }^{9}$ Since the classic problem with Parkinson's Disease is a lack in dopamine, levodopa helps to alleviate that issue. However, since levodopa comes with a plethora of side effects, this treatment is oftentimes paired with carbidopa. ${ }^{10}$ Not only does carbidopa help combat the side effects of levodopa, but it also prevents levodopa from converting into dopamine prematurely in the bloodstream; it will stagger that conversion so that it is converted to dopamine in the brain. ${ }^{10}$ Since the root of the issue of Parkinson's is a lack of dopaminergic activity in the brain, dopamine agonists also play an important role in medicinal interventions for Parkinson's disease. Instead of the converted dopamine from levodopa filling receptors, an agonist that imitates dopamine, can be used to treat symptoms.

\section{Patient outlooks}

The aim of most therapies geared towards patients with Parkinson's disease is to maximize "On" time where the therapeutic intervention is working to treat the treatment and minimizing "Off" time where the intervention expires and the symptoms return. ${ }^{9}$ However, some high doses can induce an episode of dyskinesia (Figure 1). In one randomized pairs trial they compared patient outcomes between patients with DBS and medical management with patients who used only medical management of their symptoms to manage their PD symptoms. ${ }^{11}$ Patients with both types of intervention had a better quality of life after DBS and medical management. ${ }^{9}$ This study alone demonstrates that DBS is an effective therapy to combat "Off" time for patients with PD. In a different follow up study, researchers explored whether these benefits diminish soon after the surgery, or whether they remain effective for a longer amount of time. ${ }^{12}$ In that study that looked at 69 patients who either had DBS in their subthalamic nucleus or their globus pallidus internus, they saw that the reduced "Off" time was significantly reduced for at least 3-4 years after DBS intervention. ${ }^{12}$ Overall, DBS has been proven to be an effective therapy for patients who no longer have a strong response to their medication. $^{12}$

\section{Deep brain stimulation (DBS) device features \\ DBS device components}

Deep brain stimulators typically consist of several implantable and non-implantable parts. Additionally, certain models also have the ability to be charged externally which improves the longevity of the implanted device. Implantable components consist of an implantable pulse generator (IPG) or neurostimulator (INS), leads, lead extensions, and other accessories that secure the implant in place (Skull anchor, etc.). The IPG is responsible for generating a programmable, multi-channel electrical pulse to specific parts of the brain. This pulse is propagated through leads connected to the device and stimulation occurs through specified sections of the leads called electrodes. Most currently marketed IPGs are able to support up to 2 leads with multiple electrode arrays for various programmable stimulation parameters usually supports multiple contact leads each that can be independently controlled and programed by a trained clinician (Figures 1\&2). External components typically consist of a remote control, a base station, a clinician program, and other external tools. These tools are often for support of monitoring the device functionality, battery life, and can control how the pulse is delivered to specific regions of the brain.

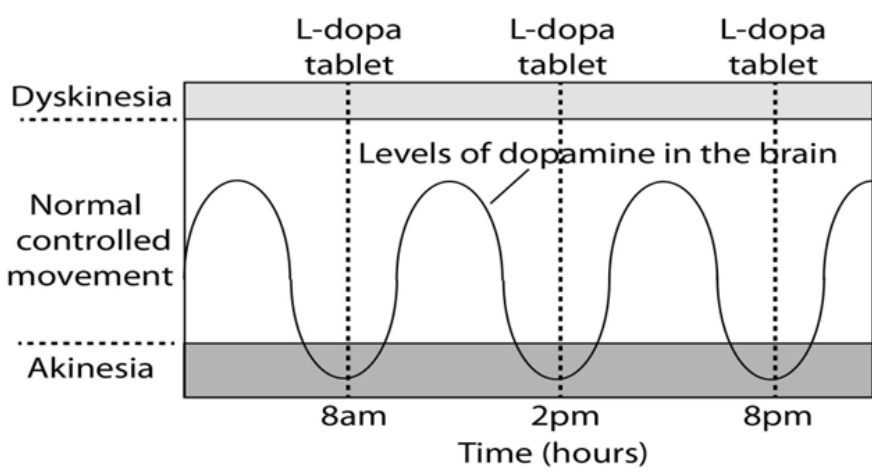

Figure I Graphic modelling the peaks and valleys of medications regimens for PD patients.

https://scienceofparkinsons.com/2020/05/08/rasgrpl/

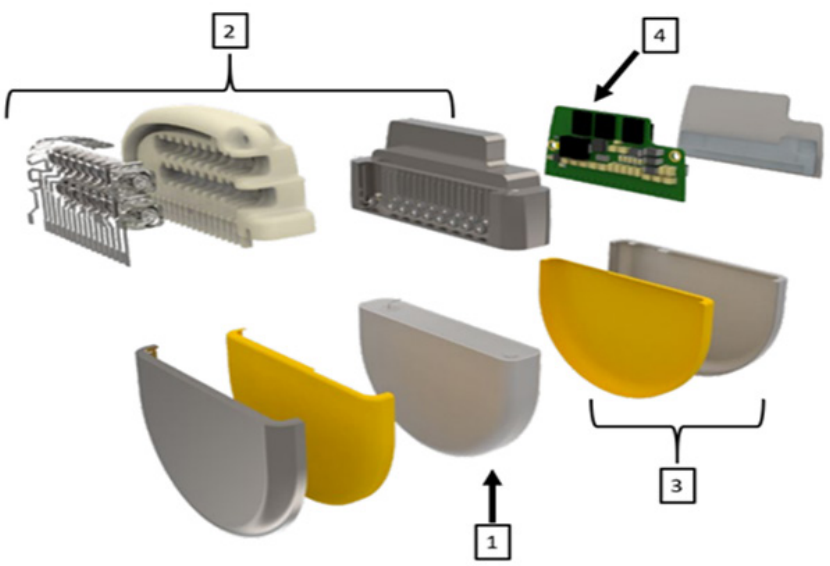

Figure 2 Exploded view of the medtronic percept IPG. parts include (I) Battery, (2) Connector assembly, (3) Casing assembly, and (4) Stimulator chipset.

\section{Deep brain stimulation for the treatment of parkinson's disease}

Prior to Deep Brain Stimulation interventions, brain lesions were a common method of surgical interventions to treat the symptoms of PD. ${ }^{4}$ Even though this was an effective method, complications were not reversible. ${ }^{4}$ Today, Deep Brain Stimulation is a surgical intervention that is less invasive than lesion surgeries, and can be reversed. This procedure is recommended for patients who cannot manage their symptoms with medication, alone. ${ }^{1}$ Deep Brain Stimulation stimulates different regions of the brain which disrupts the abnormal brain activity. Magnetic resonance imaging (MRI) is used to locate which part of the brain that the electrodes need to be placed in; these regions are typically the thalamus, subthalamic nucleus, and the globus pallidus. ${ }^{1}$ The type of symptom that is targeted will determine which region of the brain that will be manipulated. The electrodes are connected via an extension to the battery pack, which is implanted in the skin and the user can control when the device is on and off. Even though this intervention helps to alleviate some symptoms, it does not cure the disease nor stop the progression of the disease. As with many invasive procedures there are some risks associated with this treatment. ${ }^{1}$ The obvious risk is the risk of infection, while the other concern is that the patient may lose the benefits of DBS rapidly. ${ }^{1}$ 
Typically, the effects of this treatment last for about five years post surgery. ${ }^{1}$

\section{Advancements in DBS}

\begin{tabular}{ll}
\hline New developments & Description \\
\hline Closed-loop DBS & $\begin{array}{l}\text { Adaptive stimulation that responds } \\
\text { to brain activity } \\
\text { Patient specific treatments } \\
\text { Slectrical Current Shaping }\end{array}$ \\
& $\begin{array}{l}\text { Current steering } \\
\text { location specific stimulation to } \\
\text { reduce undesired areas }\end{array}$ \\
& New lead design \\
& Direction leads \\
\hline
\end{tabular}

Most advances in modern DBS devices are leading toward adaptive closed feedback loops which allow for brain activity to drive stimulation pulse on the device. Currently, DBS devices are approved to operate in an open loop system where stimulation occurs continuously and/or amplitude can be controlled by an external accessory in order to provide more or less stimulation. A closed-loop system design would allow for the IPG to only be active or respond with stimulation when abnormal brain activity is detected. In theory, this would create a longer lasting device that is capable of provide targeted treatment only when needed. This opens the potential for more efficacy for long term treatment of motor disorders.

\section{Conclusion}

This adaptive technology will first need to determine what is the threshold of abnormal activity in which to deliver stimulation. Modern DBS devices now come with phone applications that allow users to record their only activities along with providing critical information about their device from their smartphone. This can provide an easy interface for physicians to use to manipulate and monitor the DBS device (Figure 3). These features along with the ability to record brain activity in new DBS devices could bring about a new paradigm in how motor disorders are treated. Though this potentially opens a new realm of regulatory burden for manufacturers to show effectiveness of the device.

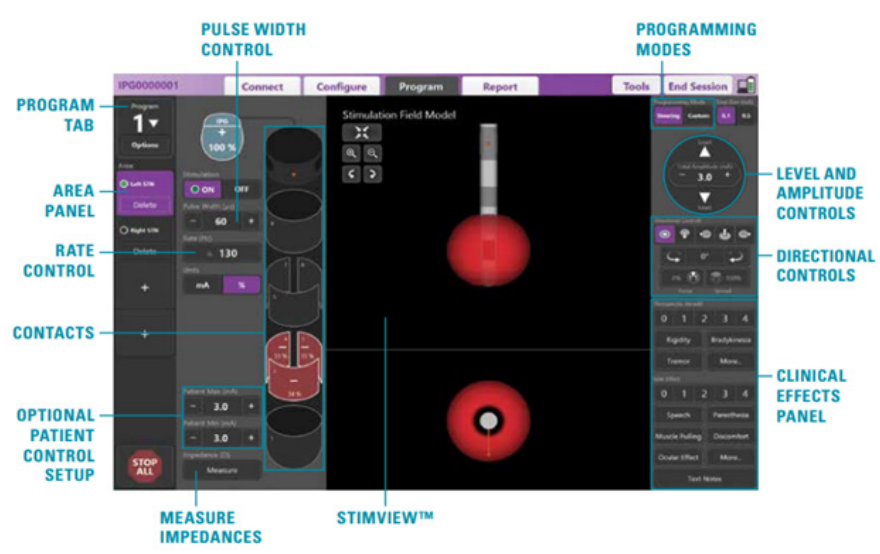

Figure 3 Example programming individual leads with the software interface of the clinical programmer. Individual contacts can be controlled by the physician to provide targeted treatment.

https://www.bostonscientific.com/en-US/medical-specialties/neurologicalsurgery/dbs/vercise-directional-dbs-system.html

\section{Acknowledgments}

None.

\section{Conflicts of interest}

Authors declare that there is no conflict of interest.

\section{Funding}

California State University Channel Islands: Extended University. Department of Biotechnology, Biomedical Engineering Emphasis. One University Drive, Camarillo, California 93012.

\section{Refernecs}

1. Butson CR, McIntyre CC. Role of electrode design on the volume of tissue activated during deep brain stimulation. J Neural Eng. 2005;3(1):1-8.

2. Butson CR, McIntyre CC. Tissue and electrode capacitance reduce neural activation volumes during deep brain stimulation. Clinical Neurophsiology. 2005;116.

3. Jan Vesper, Gregor Bara, Stefan Schu, et al. Modelling Volume of Tissue Activated for Deep Brain Stimulation. Brain Stimulation. 2015;8(2).

4. Hauser R, Deckers F, Lehert P. Parkinson's Disease Home Diary: Futher Validation and Implications for Clinical Trials. Mov Disord. 2004;19(12):1409-1413.

5. Fahn S, Elton R, Members of the UPDRS Development Committee. Unified Parkinson's Disease Rating Scale. in Recent Developments in Parkinson's Disease. NJ: MacMillan Healthcare Information. 1987. p. 153-163.

6. Peto V, Jenkinson C, Fitzpatrick R, et al. The development and validation of a short measure of functioning and well being for individuals with Parkinson's disease. Qual Life Res. 1995;4(3):241-2488.

7. Ware J, Kosinski M, Keller S, et al. SF-36 Physical and Mental Health Summary Scales: A User's manual, Boston: The Health Insitute, New England Medical Center.1994.

8. William Guy, ECDEU Assessment Manual for Psychopharmacology Revised, Rockville, MD: National Institute of Mental Health, 1976.

9. Christopher G Goetz, Werner Poewe, Olivier Rascol, et al. Movement Disorder Society Task Force report on the Hoehn and Yahr staging scale: Status and recommendations. Mov Disord. 2004;19(9):1020-1028.

10. Jurica P, Leiten C, Mattis S. Dementia Rating Scale-2. Lutz, FL: Psychological Assessments Resources. 2001.

11. Beck A, Gregory K, Steer R, et al. Beck Depression Inventory-II (BDIII), San Antonio, TX: The Psychological Corporation. 1996.

12. Leng Wendy W, Chancellor Michael B. How sacral nerve stimulation neuromodulation works. Urologic Clinics of North America. 2005;32(1):11-18. 